\title{
Sea surface topography reconstruction from $X$-band radar images
}

\author{
F. Serafino ${ }^{1}$, C. Lugni $^{2}$, and F. Soldovieri ${ }^{1}$ \\ ${ }^{1}$ Institute for Electromagnetic Sensing of the Environment, CNR, National Research Council, Via Diocleziano 328, Napoli, \\ 80124 Italy \\ ${ }^{2}$ INSEAN, the Italian Ship Model Basin, Department of Seakeeping and Maneuverability, Via di Vallerano, 139, Roma, \\ 00128 Italy
}

Received: 21 August 2008 - Revised: 30 October 2008 - Accepted: 30 October 2008 - Published: 14 November 2008

\begin{abstract}
The paper deals with the feasibility study of the sea state monitoring starting from X-band radar images. The exploitation of radar images allows to achieve a global vision of the sea state compared to the local vision given by the usual sensors as the buoys. The processing approach is based on the formulation of problem as an inverse one where starting from the electromagnetic field backscattered by the sea surface, the information about the sea state are retrieved. The reliability of the inversion procedure is shown by processing synthetic and experimental data where particular attention is focussed to the determination of the sea current and speed of the vessel.
\end{abstract}

\section{Introduction}

In recent years, the exploitation of the remote sensing observations based on radar systems for sea state monitoring has gained an increasing interest. In particular, radar systems allocated on ships and platforms allow one to assess, in real time and with high accuracy, the elevation of the sea waves and other parameters that characterize the sea state (Young et al., 1985; Ziemer et al., 1991; Nieto Borge et al., 2004; Nieto Borge and Guedes Soares, 2000; Nieto Borge, 1997; Hessner et al.,1985).

From the point of view of the "physics", to gain such an information it is made possible by the electromagnetic interaction between the electromagnetic field radiated by a radar and the capillary waves on the surface of the sea (Plant and Keller, 1990; Wenzel, 1990). Such an interaction, due to the phenomenon of resonance Bragg, arises a backscattered electromagnetic field that is modulated by the longer waves; accordingly, such a backscattered field, which is re- ceived by the same radar, carries out information about the sea waves. More in detail, the electromagnetic field modulation is a nonlinear process due to three different causes: hydrodynamic modulation (HM), tilt modulation (TM), and shadowing ( $\mathrm{SH}$ ). HM describes the modulation of the energy of the ripples by the interaction with the longer waves; TM is the modulation due to the variation of the angle under which the sea wave surface is viewed by the radar; $\mathrm{SH}$ accounts for the electromagnetic shadowing of the sea surface by higher waves (Plant and Keller, 1990; Wenzel, 1990). Due to these modulation effects, the radar image is not a "direct representation" of the sea state and thus the need of processing approaches arises with he aim of extracting the information about the sea surface state starting form the backscattered field.

In particular, data processing consists of an inversion procedure that, starting from the X-band radar images sequences, provides an estimation of the elevation of the sea surface meant as a function of the time and the spatial variables of the sea zone illuminated by the radar (Nieto Borge et al., 2004).

In this framework, here we will present the results of the inversion approach, first for the case of simulated data and then for realistic measurements acquired from a radar located on a moving ship. In particular, for the realistic case, attention is focussed to the estimation of the marine surface current whose value is a decisive factor in ensuring the reliability of the overall monitoring. In particular, the estimation of the surface current has been performed by a strategy based on a "correlation" procedure between the measured signal and the theoretical model based on the dispersion relation accounting for the sea-state (Serafino et al., 2008).

\section{Correspondence to: F. Soldovieri}

(soldovieri.f@irea.cnr.it)

Published by Copernicus Publications on behalf of the European Geosciences Union. 


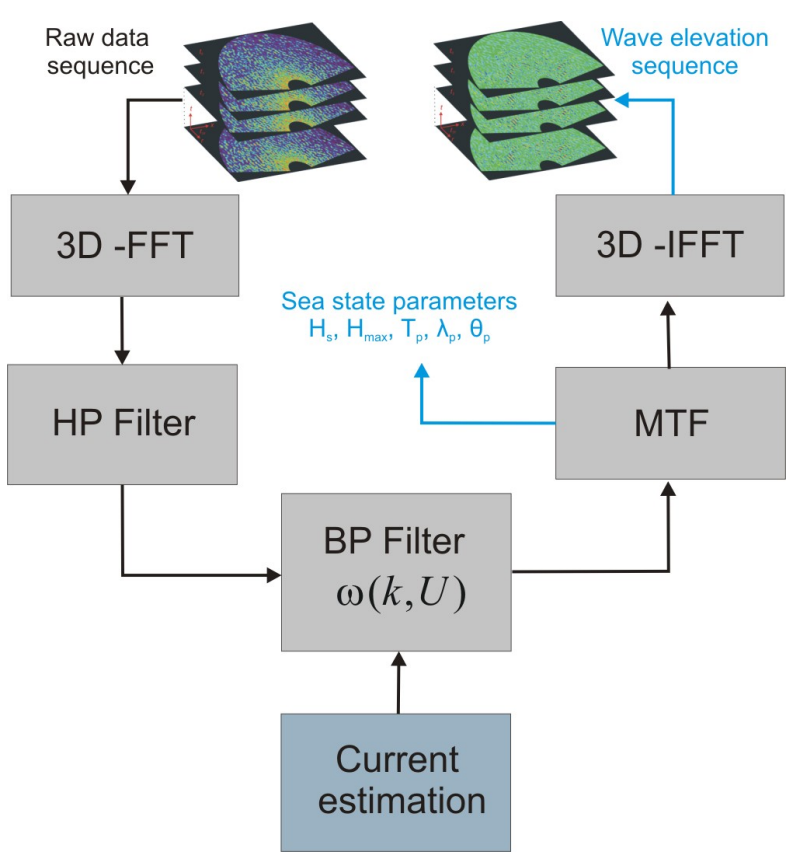

Fig. 1. Block diagram of the inversion procedure.

\section{Processing scheme}

The flow-chart of the inversion scheme is depicted in Fig. 1 and briefly detailed in (Nieto Borge et al., 2004; Serafino et al., 2008). First, the 3-D Fourier Transform of the space-time radar images sequence is computed; the second step consists in a spectral filtering via a dispersion relation in order to select the frequencies associated to the linear gravity waves that is the phenomenon of interest for the problem at hand (Nieto Borge et al., 2004; Serafino et al., 2008); then, the filtered radar spectrum is transformed to the sea spectrum by the Modulation Transfer Function (MTF) (Nieto Borge et al., 2004); finally, the filtered and modulated spectrum is Fourier transformed to provide the sea state images in the space-time domain.

The second step, i.e., the spectral filtering one, has the crucial effect on the quality of the overall inversion procedure and the reliability of such a step is based on the use of the right dispersion relation (Serafino et al., 2008). The exploitation of the correct dispersion relation depends on the sea surface current intensity. In fact, when the surface currents are not correctly determined and/or accounted for in the dispersion relation, the results of the overall inversion are quite poor. This difficulty is further increased when the values of the surface current become high or if the data are acquired by moving ships, since in these cases the problem of the determination of the current is quite complicated and in addition particular attention has to be made to the filtering procedure.

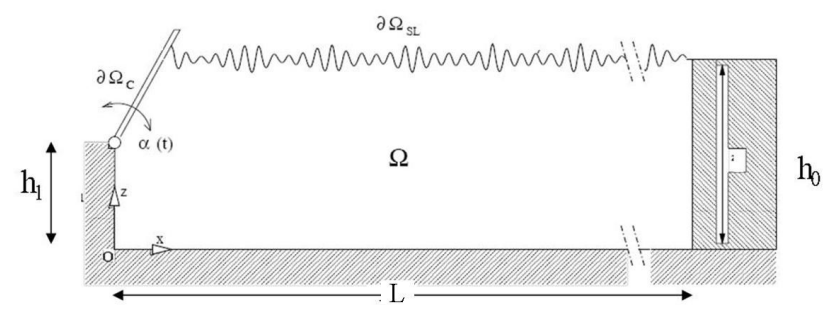

Fig. 2. Pictorial sketch of the mathematical problem.

Table 1. Parameters description of Fig. 2

\begin{tabular}{lll}
\hline$h_{0}(\mathrm{~m})$ & $h_{1}(\mathrm{~m})$ & $\mathrm{L}(\mathrm{m})$ \\
\hline 3.6 & 1.8 & 230 \\
\hline
\end{tabular}

The measure of the sea surface currents is not typically available by independent observations and then such an information has to be estimated directly from the radar data. In the Section below, we will present the results about the estimation of the surface current achieved by the method presented in (Serafino et al., 208) based on the "correlation" between the measured spectrum and the characteristic function having as a support the one of the dispersion relation.

\section{Simulated data and numerical result}

The inversion procedure presented in the Section above has been firstly applied to synthetic data.

A 2-D numerical wavemaker (Lugni, 1999) has been exploited to reproduce the physical conditions existing in a real wave tank (see Fig. 2) with the values of the geometrical parameters that are given in Table 1. The spatial and time evolution of a free-surface $\partial \Omega_{S L}$ wave train, generated through a hinged paddle $\partial \Omega_{c}$ moving with angular velocity $\dot{\alpha}(t)$, has been investigated by an non viscid model. In this framework the mathematical statement is described through the Laplace equation for the velocity potential function. Once the velocity potential is computed on the boundary domain, the nonlinear free-surface equations are stepped forward by a fourthorder Runge-Kutta scheme and the motion of the wavemaker is updated.

To save computational time and memory for the simulation of long time wave evolution, a domain decomposition technique has been used (Wang et al., 1995). Further details of the numerical model used as well as of the treatment of the free-surface can be found in Lugni (1999).

A JONSWAP sea spectrum with $H_{1 / 3}=8.8 \mathrm{~cm}$ and $T_{0}=1.967 \mathrm{~s}$ has been reproduced in the numerical wave tank for the present case. A factor scale 20 has been used to reconstruct the wave elevations corresponding to the full scale 

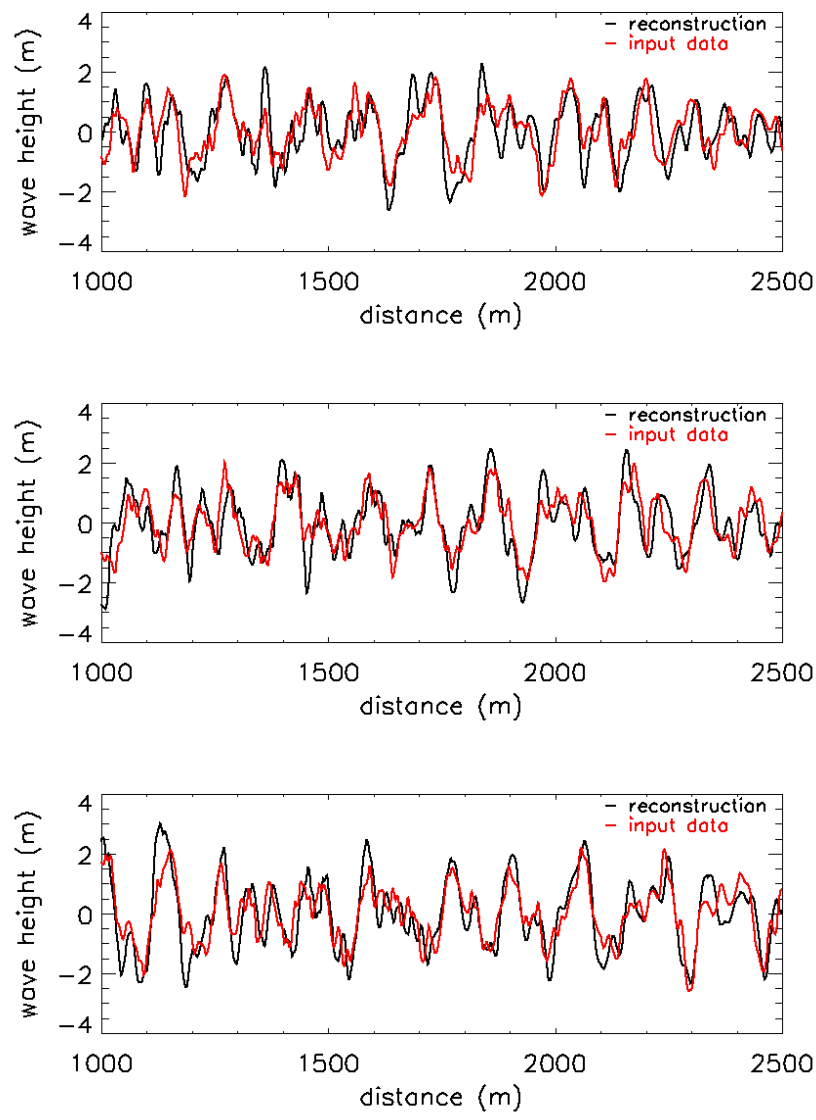

Fig. 3. Comparison between the reconstruction (black) of the wave height and the simulated input data sequences (red). From top to bottom the images refer to: $36 \mathrm{~s}, 55 \mathrm{~s}$ and $78 \mathrm{~s}$ with respect to the start time, respectively.

sea state SS4 $\left(H_{1 / 3}=1.76 \mathrm{~m}\right.$ and $\left.T_{0}=8.8 \mathrm{~s}\right)$. Here $H_{1 / 3}$ represents the significant wave height, and $T_{0}$ the modal period associated with the prescribed spectrum.

After the generation of the simulated waves, the radar data have been simulated by the procedure in (Nieto Borge et al., 2004); in particular, we have considered the two most important modulation effects such as the shadowing $(\mathrm{SH})$ and the tilt (TM) ones.

Finally, the radar data have been processed by the inversion procedure presented in Sect. 2. Figure 3 shows the reconstruction of the wave height compared with the input data sequences, referred to the following time instants: $36 \mathrm{~s}, 55 \mathrm{~s}$ and $78 \mathrm{~s}$, respectively.

To implement a statistical analysis of the result provided by the inversion procedure we have computed and compared the reconstructed significant wave value and the modal period with the ones of the input data. The obtained values are:

$$
\begin{aligned}
& H_{1 / 3}=1.76 \mathrm{~m} \text { referred to the simulated input data, } \\
& H_{1 / 3}=1.75 \mathrm{~m} \text { referred to the reconstruction, }
\end{aligned}
$$
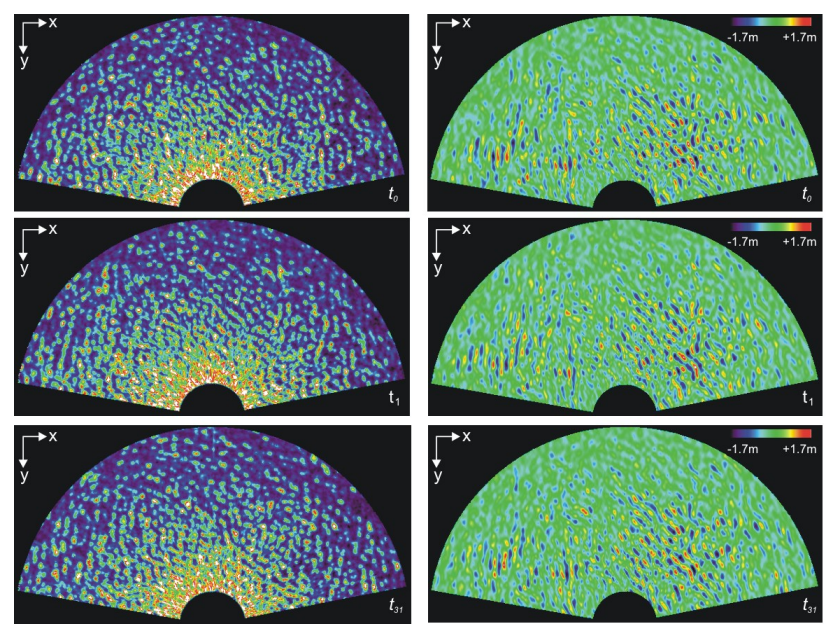

Fig. 4. Left: Digitized image sequence (form top to bottom: first, second and last images, respectively) of the radar data with. Right reconstructed wave height by the inversion algorithm.

$T_{0}=8.8 \mathrm{~s}$ referred to the simulated input data,

$T_{0}=9.0 \mathrm{~s}$ referred to the reconstruction.

and the estimated parameters are almost coincident demonstrating the high accuracy of the inversion technique.

\section{Radar system and real data processing}

The processing chain is then applied to experimental data.

The system used for acquiring and storing the data is the WAVEX (Marine Radar Wave Extractor) system. This system provides real time information on sea state parameters starting by images acquired by a conventional marine $\mathrm{X}$-band radar mounted on a moving vessel The raw data consist of a sequence of 32 images, each one made up of $600 \times 600$ pixels. The total extent of each image is about $10 \mathrm{~km}^{2}$ while time span corresponding to a complete acquisition is $76.8 \mathrm{~s}$.

Figure 4 shows a sequence (first two and last one of 32) of raw data images recorded on 22 November 2007 in the area of the Mediterranean sea between Malta and Sicily islands. In particular, the proposed method estimated an equivalent surface current of $8.2 \mathrm{~m} / \mathrm{s}$ that accounts also for the vessel speed provided by GPS measurement and equal to about $8 \mathrm{~m} / \mathrm{s}$.

\section{Conclusions}

This work has dealt with the problem of the sea state parameters monitoring by starting from marine radar images acquired by a fixed platform or a moving ship. In particular, the problem has been stated as an inverse one and the solution approach is based on different steps mainly related to 
Fourier transform and filtering procedures. One of the crucial factors affecting the filtering procedures is the surface current intensity that has to be estimated by the same radar data.

In this paper, we have presented the results achieved by a novel strategy to determine the surface current. The effectiveness of the overall reconstruction procedure has been validated first with synthetic data, generated by a 2-D wave maker, and then thanks to realistic radar data collected by a moving vessel.

Edited by: L. V. Eppelbaum

Reviewed by: two anonymous referees

\section{References}

Hessner K., Reichert K., Dittmer J., Nieto Borge J. C., and Gunther H.: Evaluation of WaMoS II wave data, in : Proc. Fourth Int. Symp. on Ocean Wave Measurement and Analysis, San Francisco, USA, 221-230, 2000.

Lugni, C.: An investigation on the interaction between free-surface waves and a marine structure (in Italian), PhD Thesis on Applied and Theoretical Mechanics, University of Rome "La Sapienza", 1999.

Nieto Borge J. C.: Analisis de Campos de Oleaje Mediante Radar de Navegacion en Banda X, Ph.D. Thesis at the Dep. of Physics of the University of Madrid, 1997.
Nieto Borge J. C. and Guedes Soares C.: Analysis of directional wave fields using X-Band navigation radar, Coastal Eng., 40, 375-391, 2000.

Nieto Borge J. C., Rodriguez R. G., Hessner K., and Gonzales I. P.: Inversion of marine radar images for surface wave analysis, J. Atmos. Ocean. Technol., 21, 1291-1300, 2004.

Plant W. J. and Keller W. C.: Evidence of Bragg scattering in microwave Doppler spectra of sea return, J. Geophys. Res., 95, 16299-16310, 1990.

Serafino F., Lugni C., and Soldovieri F.: Validation of a reconstruction approach for sea-state monitoring from marine radar data on moving ships, IEEE Geoacience and Remote sensing Letters, accepted, 2008.

Wang P., Yao Y., and Tulin M. P.: An efficient numerical wave tank for nonlinear water waves, based on the multi-subdomain approach with BEM, Int. Journal for Numer. Meth. in Fluids, 20, 1315-1336, 1995.

Wenzel L. B.: Electromagnetic scattering from the sea at low grazing angles, Surface Waves and Fluxes, edited by: Geernaert, G. L. and Plant, W. J., Kluwer Academic, 41-108, 1990.

Young R., Rosenthal W., and Ziemer F.: Three-dimensional analysis of marine radar images for the determination of ocean wave directionality and surface currents, J. Geophys. Res., 90, 10491059, 1985.

Ziemer F. and Rosenthal W.: Directional spectra from shipboard navigation radar during LEWEX, Directional Ocean Wave Spectra: Measuring, Modeling, Predicting, and Applying, R. C. Beal, Ed., The Johns Hopkins University Press, 125-127, 1991. 